MATHEMATICS OF COMPUTATION

Volume 66, Number 219, July 1997, Pages 1185-1194

S 0025-5718(97)00863-6

\title{
COMPUTATION OF RELATIVE CLASS NUMBERS OF CM-FIELDS
}

\author{
STÉPHANE LOUBOUTIN
}

\begin{abstract}
It was well known that it is easy to compute relative class numbers of abelian CM-fields by using generalized Bernoulli numbers (see Theorem 4.17 in Introduction to cyclotomic fields by L. C. Washington, Grad. Texts in Math., vol. 83, Springer-Verlag, 1982). Here, we provide a technique for computing the relative class number of any CM-field.
\end{abstract}

\section{Statement of the Results}

Proposition 1. Let $n \geq 1$ be an integer and $\alpha>1$ be real. Set $P_{n}(x)=\sum_{k=0}^{n-1} \frac{1}{k !} x^{k}$,

$$
f_{n}(s)=\Gamma^{n}(s) A^{-2 s}\left(\frac{1}{2 s-1}+\frac{1}{2 s-2}\right)
$$

and

$$
K_{n}(A)=\frac{A^{2}}{i \pi} \int_{\alpha-i \infty}^{\alpha+i \infty} f_{n}(s) d s
$$

Then, it holds

$$
0 \leq K_{n}(A) \leq 2 P_{n}\left(n A^{2 / n}\right) e^{-n A^{2 / n}} \leq 2 n \exp \left(-A^{2 / n}\right) .
$$

Theorem 2. Let $\mathbf{N}$ be a totally imaginary number field of degree $2 n$ which is a quadratic extension of a totally real number field $\mathbf{N}^{+}$of degree $n$, i.e. $\mathbf{N}$ is a CMfield. Let $w_{\mathbf{N}}$ be the number of roots of unity in $\mathbf{N}, Q_{\mathbf{N}} \in\{1,2\}$ be the Hasse unit index of $\mathbf{N}$, and $d_{\mathbf{N}}, \zeta_{\mathbf{N}}$ and $d_{\mathbf{N}^{+}}, \zeta_{\mathbf{N}^{+}}$be the absolute values of the discriminants and the Dedekind zeta functions of $\mathbf{N}$ and $\mathbf{N}^{+}$, respectively. Let $\chi_{\mathbf{N} / \mathbf{N}^{+}}$be the quadratic character assocciated with the quadratic extension $\mathbf{N} / \mathbf{N}^{+}$and let $\phi_{k}$ be the coefficients of the Dirichlet series $\left(\zeta_{\mathbf{N}} / \zeta_{\mathbf{N}^{+}}\right)(s)=L\left(s, \chi_{\mathbf{N} / \mathbf{N}^{+}}\right)=\sum_{k \geq 1} \phi_{k} k^{-s}$, $\Re(s)>1$. Set $A_{\mathbf{N} / \mathbf{N}^{+}}=\sqrt{d_{\mathbf{N}} / \pi^{n} d_{\mathbf{N}^{+}}}$.

We have

$$
h_{\mathbf{N}}^{-}=\frac{Q_{\mathbf{N}} w_{\mathbf{N}}}{(2 \pi)^{n}} \sqrt{\frac{d_{\mathbf{N}}}{d_{\mathbf{N}^{+}}}} \sum_{k \geq 1} \frac{\phi_{k}}{k} K_{n}\left(k / A_{\mathbf{N} / \mathbf{N}^{+}}\right),
$$

and according to (3) this series (4) is absolutely convergent. Moreover, set

$$
B(\mathbf{N}) \stackrel{\text { def }}{=} A_{\mathbf{N} / \mathbf{N}^{+}}\left(\frac{\lambda}{n} \log A_{\mathbf{N} / \mathbf{N}^{+}}\right)^{n / 2} .
$$

Received by the editor December 5, 1995 and, in revised form, April 12, 1996.

1991 Mathematics Subject Classification. Primary 11R29, 11Y35; Secondary 11R42.

(C)1997 American Mathematical Society 
Then, if $\lambda>1$ and $n$ are given, then the limit of $\left|h_{\mathbf{N}}^{-}-h_{\mathbf{N}}^{-}(M)\right|$ as $A_{\mathbf{N}} / \mathbf{N}^{+}$approaches infinity is equal to 0 , where $h_{\mathbf{N}}^{-}(M)$ is the approximation of the relative class number obtained by disregarding in the series occurring in (4) the indices $k>M \geq B(\mathbf{N})$.

For example, if $\mathbf{N}$ of degree $m=2 n$ is the narrow Hilbert class field of a real quadratic number field $\mathbf{L}$ of discriminant $d_{\mathbf{L}}$, we have

$$
B(\mathbf{N})=\left(\frac{\lambda}{4 \pi}\right)^{m / 4} d_{\mathbf{L}}^{m / 8} \log ^{m / 4}\left(d_{\mathbf{L}} / \pi^{2}\right) .
$$

The following Proposition 3 explains how we compute the numerical values of the function $A \mapsto K_{n}(A)$ according to its series expansion:

Proposition 3. Take $A>0$. It holds

$$
K_{n}(A)=1+\pi^{n / 2} A+2 A^{2} \sum_{m \geq 0} \operatorname{Res}_{s=-m}\left(f_{n}\right) .
$$

This series is absolutely convergent and for any integer $M \geq 0$ we have

$$
\left|2 A^{2} \sum_{m>M} \operatorname{Res}_{s=-m}\left(f_{n}\right)\right| \leq \frac{\pi^{n / 2} A^{2 M+3}}{(M+1)(M ! / 2)^{n}} .
$$

Finally, the following Proposition 4 explains how to compute recursively the values of the residues $\operatorname{Res}_{s=-m}\left(f_{n}\right)$ occurring in (6):

Proposition 4. We have ${ }^{1}$

$$
\operatorname{Res}_{s=-m}\left(f_{n}\right)=-(-1)^{n m} \frac{A^{2 m}}{(m !)^{n}} \sum_{i=-n}^{-1} 2^{-1-i} h_{i}(m)\left((2 m+1)^{i}+(2 m+2)^{i}\right)
$$

where the $h_{i}(m)$ 's are computed recursively from the $h_{i}(0)$ 's by using

$$
h_{i}(m+1)=\sum_{j=-n}^{i} h_{j}(m) \frac{b_{i-j}}{(m+1)^{i-j}} \quad \text { and } \quad \sum_{j=-n}^{-1} h_{j}(0) s^{j}+O(1)=\Gamma^{n}(s) A^{-2 s},
$$

where $b_{k}=C_{n+k-1}^{n-1}=((k+n-1) ! / k !(n-1) !)$. Thus, if

$$
\Gamma^{n}(s+1)=\sum_{i=0}^{n-1} h_{i} s^{i}+O\left(s^{n}\right)
$$

then

$$
h_{j-n}(0)=\sum_{i=0}^{j} \frac{(-2 \log A)^{i}}{i !} h_{j-i} \quad(0 \leq j \leq n-1) .
$$

For proving these results, obvious questions of convergence of series and integrals, and questions of inversions of integrals and summations will not be gone into.

\footnotetext{
${ }^{1}$ Note the misprint in the formula given in [Lou 2].
} 


\section{INTRODUCTION}

Prior to the method we have developed here, the only general method for computing the relative class number of any CM-field was that developed by T. Shintani (see [Oka 1] and [Oka 2] for examples of actual relative class number computations using Shintani's ideas). However, his method requires the knowledge of a great deal of information on the maximal totally real subfield $\mathbf{N}^{+}$. In particular, it requires the knowledge of a system of fundamental units of the group of totally positive units of $\mathbf{N}^{+}$. However, what makes the concept of CM-field an attractive one is that the relative class number formula

$$
h_{\mathbf{N}}^{-}=\frac{Q_{\mathbf{N}} w_{\mathbf{N}}}{(2 \pi)^{n}} \sqrt{\frac{d_{\mathbf{N}}}{d_{\mathbf{N}^{+}}}} \frac{\operatorname{Res}_{s=1}\left(\zeta_{\mathbf{N}}\right)}{\operatorname{Res}_{s=1}\left(\zeta_{\mathbf{N}^{+}}\right)}=\frac{Q_{\mathbf{N}} w_{\mathbf{N}}}{(2 \pi)^{n}}\left(\sqrt{\frac{d_{\mathbf{N}}}{d_{\mathbf{N}^{+}}}}\right) L\left(1, \chi_{\mathbf{N} / \mathbf{N}^{+}}\right)
$$

enables us to get lower bounds on relative class numbers and solve class number and class group problems for CM-fields precisely because (12) does not involve any regulator (see [Lou-Oka] and [LOO]). Thus, the reader may possibly feel dissatisfied that he should have to know beforehand a good grasp of the unit group of $\mathbf{N}^{+}$before he can compute $h_{\mathbf{N}}^{-}$, whereas (12) gives an expression for $h_{\mathbf{N}}^{-}$which does not involve units. The reader may now possibly feel satisfied that this paper shows how using (12) he indeed gets an efficient method for computing $h_{\mathbf{N}}^{-}$provided that he only knows how to compute the decomposition of any rational prime into a product of prime ideals of $\mathbf{N}$. The key point of our method is to establish the holomorphic continuation of $s \mapsto\left(\zeta_{\mathbf{N}} / \zeta_{\mathbf{N}^{+}}\right)(s)=L\left(s, \chi_{\mathbf{N} / \mathbf{N}^{+}}\right)$in the same way Riemann did in the case of the Riemann zeta function (by using Mellin transformation) and to evaluate the resulting series at $s=1$ (see section 4).

Finally, we note that the results of this paper are better than those of [Lou 3]. Indeed, $B(\mathbf{N})$ in (5) is $n^{n / 2}$-fold better than the one we gave in [Lou 3]. Moreover, our proof of (3) (in section 3) is more satisfactory and elegant than the one we gave in [Lou 3].

\section{Proof of Proposition 1}

We use:

Lemma 5. Let $\alpha>1$ be real. We have

$\int_{\alpha-i \infty}^{\alpha+i \infty} u^{s} \frac{d s}{2 s-1}=\left\{\begin{array}{ll}0 & \text { if } u<1, \\ i \pi \sqrt{u} & \text { if } u>1 ;\end{array} \quad\right.$ and $\quad \int_{\alpha-i \infty}^{\alpha+i \infty} u^{s} \frac{d s}{2 s-2}= \begin{cases}0 & \text { if } u<1 \\ i \pi u & \text { if } u>1\end{cases}$

Now, using

$$
\Gamma^{n}(s)=\iint e^{-\operatorname{Tr}(y)} y^{s} \frac{d y}{y}
$$

where the multiple integral ranges over $\left(y_{1}, \cdots, y_{n}\right) \in\left(\mathbf{R}_{+}^{*}\right)^{n}$ and where we set $y=y_{1} y_{2} \cdots y_{n}$ and $\operatorname{Tr}(y)=y_{1}+y_{2}+\cdots+y_{n}$, leads to

$$
K_{n}(A)=\frac{A^{2}}{i \pi} \iint e^{-\operatorname{Tr}(y)}\left(\int_{\alpha-i \infty}^{\alpha+i \infty}\left(y / A^{2}\right)\left(\frac{1}{2 s-1}+\frac{1}{2 s-2}\right)\right) \frac{d y}{y} .
$$


Using Lemma 5 yields

$$
K_{n}(A)=A^{2} \iint_{y \geq A^{2}}\left(\sqrt{y / A^{2}}+\left(y / A^{2}\right)\right) e^{-\operatorname{Tr}(y)} \frac{d y}{y} \leq 2 \iint_{y \geq A^{2}} e^{-\operatorname{Tr}(y)} d y .
$$

For example, we get $K_{1}(A) \leq 2 e^{-A^{2}}$. Now, using the arithmetic-geometric mean inequality yields that $\left\{\left(y_{1}, \cdots, y_{n}\right) ; y \geq A^{2}\right\}$ is included in $\left\{\left(y_{1}, \cdots, y_{n}\right) ; \operatorname{Tr}(y) \geq\right.$ $\left.n A^{2 / n}\right\}$, which yields

$$
K_{n}(A) \leq 2 \iint_{\operatorname{Tr}(y) \geq n A^{2 / n}} e^{-\operatorname{Tr}(y)} d y .
$$

Then, the following easily proved Lemma 6 provides us with the desired result. We finally notice that we get a shorter and more satisfactory proof of [Lou 3, Proposition 1]:

Lemma 6. Set $P_{n}(x)=\sum_{k=0}^{n-1} x^{k} / k$ !. Then

$$
P_{n}(\alpha) e^{-\alpha}=\iint_{\substack{\left(y_{1}, \cdots, y_{n}\right) \in \mathbf{R}_{+}^{*} \\ \operatorname{Tr}(y) \geq \alpha}} e^{-\operatorname{Tr}(y)} d y \leq n \int_{\alpha / n}^{+\infty} e^{-y} d y=n e^{-\alpha / n} .
$$

Proof. Use

$$
\begin{aligned}
& \left\{\left(y_{1}, \cdots, y_{n}\right) \in \mathbf{R}_{+}^{*}, \operatorname{Tr}(y) \geq \alpha\right\} \\
& \quad \subseteq \bigcup_{i=1}^{n}\left\{\left(y_{1}, \cdots, y_{n}\right), y_{i} \geq \frac{\alpha}{n} \text { and } y_{j} \geq 0 \text { for } j \neq i\right\} .
\end{aligned}
$$

\section{Proof of Theorem 2}

Let $\mathbf{K}$ be a number field of degree $n=r_{1}+2 r_{2}$, where $r_{1}$ is the number of real places of $\mathbf{K}$ and $r_{2}$ the number of complex places of $\mathbf{K}$. Let $\zeta_{\mathbf{K}}$ and $\operatorname{Reg}_{\mathbf{K}}$ be the Dedekind zeta function and regulator of $\mathbf{K}$. We set

$$
\begin{aligned}
& A_{\mathbf{K}}=2^{-r_{2}} d_{\mathbf{K}}^{1 / 2} \pi^{-}\left(r_{1}+2 r_{2}\right) / 2 \\
& \lambda_{\mathbf{K}}=\frac{2^{r_{1}} h_{\mathbf{K}} \operatorname{Reg}_{\mathbf{K}}}{w_{\mathbf{K}}} \text { where } w_{\mathbf{K}} \text { is the number of roots of unity in } \mathbf{K}, \\
& F_{\mathbf{K}}(s)=A_{\mathbf{K}}^{s} \Gamma\left(\frac{s}{2}\right)^{r_{1}} \Gamma(s)^{r_{2}} \zeta_{\mathbf{K}}(s) .
\end{aligned}
$$

Hence, $F_{\mathbf{K}}$ a a simple pole at $s=1$ with residue $\lambda_{\mathbf{K}}$, and $F_{\mathbf{K}}(s)=F_{\mathbf{K}}(1-s)$.

From now on, we let $\mathbf{N}$ be a CM-field of degree $2 n$, i.e. $\mathbf{N}$ is a totally imaginary number field of degree $2 n$ which is a quadratic extension of a totally real number field $\mathbf{N}^{+}$of degree $n$. Define the $\phi_{k}$ 's by :

$$
\Phi_{\mathbf{N} / \mathbf{N}^{+}}(s)=\frac{\zeta_{\mathbf{N}}}{\zeta_{\mathbf{N}^{+}}}(s)=\sum_{k \geq 1} \phi_{k} k^{-s} \quad(\Re(s)>1) .
$$

Then, $\left(\zeta_{\mathbf{N}} / \zeta_{\mathbf{N}^{+}}\right)(s)=L\left(s, \chi_{\mathbf{N} / \mathbf{N}^{+}}\right)$yields

$$
\phi_{k}=\sum_{N_{\mathbf{N}^{+} / \mathbf{Q}}(\mathbf{I})=k} \chi_{\mathbf{N} / \mathbf{N}^{+}}(\mathbf{I})
$$

where $\mathbf{I}$ ranges over the integral ideals of $\mathbf{N}^{+}$of norm $k$. Now,

$$
\Phi_{\mathbf{N} / \mathbf{N}^{+}}=\zeta_{\mathbf{N}} / \zeta_{\mathbf{N}^{+}} \text {and } \Psi_{\mathbf{N} / \mathbf{N}^{+}}=F_{\mathbf{N}} / F_{\mathbf{N}^{+}}
$$


are entire and $\Psi_{\mathbf{N} / \mathbf{N}^{+}}(s)=\Psi_{\mathbf{N} / \mathbf{N}^{+}}(1-s)$. Notice that

$$
\Psi_{\mathbf{N} / \mathbf{N}^{+}}(1)=\frac{\lambda_{\mathbf{N}}}{\lambda_{\mathbf{N}^{+}}}=\frac{h_{\mathbf{N}}^{-}}{Q_{\mathbf{N}} w_{\mathbf{N}}}
$$

where $Q_{\mathbf{N}} \in\{1,2\}$ is the Hasse unit index of $\mathbf{N}$ (see [Wa, Th. 4.16]). Since

$$
\Gamma(s)=\frac{2^{s-1}}{\sqrt{\pi}} \Gamma\left(\frac{s}{2}\right) \Gamma\left(\frac{s+1}{2}\right),
$$

using (13) for $\mathbf{N}$ and $\mathbf{N}^{+}$leads to

$$
\Psi_{\mathbf{N} / \mathbf{N}^{+}}(s)=c_{\mathbf{N} / \mathbf{N}^{+}} A_{\mathbf{N} / \mathbf{N}^{+}}^{s} \Gamma^{n}\left(\frac{s+1}{2}\right) \Phi_{\mathbf{N} / \mathbf{N}^{+}}(s)
$$

where

$$
c_{\mathbf{N} / \mathbf{N}^{+}}=1 /(4 \pi)^{n / 2} \text { and } A_{\mathbf{N} / \mathbf{N}^{+}}=\sqrt{d_{\mathbf{N}} / \pi^{n} d_{\mathbf{N}^{+}}} .
$$

Note that

$$
c_{\mathbf{N} / \mathbf{N}^{+}} A_{\mathbf{N} / \mathbf{N}^{+}}=\frac{1}{(2 \pi)^{n}} \sqrt{\frac{d_{\mathbf{N}}}{d_{\mathbf{N}^{+}}}} .
$$

Set

$$
\hat{\Psi}_{\mathbf{N} / \mathbf{N}^{+}}(x)=\frac{1}{2 i \pi} \int_{\alpha-i \infty}^{\alpha+i \infty} \Psi_{\mathbf{N} / \mathbf{N}^{+}}(s) x^{-s} d s \quad(\alpha>1),
$$

i.e., $\hat{\Psi}_{\mathbf{N} / \mathbf{N}^{+}}$is the Mellin transform of the function $\Psi_{\mathbf{N} / \mathbf{N}^{+}}$. Using (18) and (16) yields

$$
\hat{\Psi}_{\mathbf{N} / \mathbf{N}^{+}}(x)=c_{\mathbf{N} / \mathbf{N}^{+}} \sum_{k \geq 1} \phi_{k} H_{n}\left(k x / A_{\mathbf{N} / \mathbf{N}^{+}}\right) \quad(x>0),
$$

with

$$
\begin{aligned}
H_{n}(x) & =\frac{1}{2 i \pi} \int_{\alpha-i \infty}^{\alpha+i \infty} \Gamma^{n}\left(\frac{s+1}{2}\right) x^{-s} d s \\
& =\frac{1}{i \pi} \int_{\alpha-i \infty}^{\alpha+i \infty} \Gamma^{n}(S) x^{1-2 S} d S \quad(x>0 \text { and } \alpha>0) .
\end{aligned}
$$

Now, we move the integral (18) to the line $\Re(s)=1-\alpha$. Since $\Psi_{\mathbf{N} / \mathbf{N}^{+}}$is entire, we do not pick up any residue. Then, we use the functional equation

$$
\Psi_{\mathbf{N} / \mathbf{N}^{+}}(s)=\Psi_{\mathbf{N} / \mathbf{N}^{+}}(1-s)
$$

satisfied by $\Psi_{\mathbf{N} / \mathbf{N}^{+}}$to come back to the line $\Re(s)=\alpha$. We get

$$
x \hat{\Psi}_{\mathbf{N} / \mathbf{N}^{+}}(x)=\hat{\Psi}_{\mathbf{N} / \mathbf{N}^{+}}(1 / x) \quad(x>0) .
$$

Mellin's inversion formula and (21) yield

$$
\Psi_{\mathbf{N} / \mathbf{N}^{+}}(s)=\int_{0}^{\infty} \hat{\Psi}_{\mathbf{N} / \mathbf{N}^{+}}(x) x^{s} \frac{d x}{x}=\int_{1}^{\infty} \hat{\Psi}_{\mathbf{N} / \mathbf{N}^{+}}(x)\left\{x^{s-1}+x^{-s}\right\} d x .
$$


By using (22), (19) and (20) we thus get

$$
\begin{aligned}
& \Psi_{\mathbf{N} / \mathbf{N}^{+}}(s) \\
& =c_{\mathbf{N} / \mathbf{N}^{+}} \sum_{k \geq 1} \phi_{k} \int_{1}^{\infty}\left(\frac{1}{i \pi} \int_{\alpha-i \infty}^{\alpha+i \infty}\left(\frac{k x}{A_{\mathbf{N} / \mathbf{N}^{+}}}\right)^{1-2 S} \Gamma^{n}(S)\left\{x^{s-1}+x^{-s}\right\} d S\right) d x \\
& =c_{\mathbf{N} / \mathbf{N}^{+}} \sum_{k \geq 1} \phi_{k} \frac{1}{i \pi} \int_{\alpha-i \infty}^{\alpha+i \infty} \Gamma^{n}(S)\left(\int_{1}^{\infty}\left(\frac{k x}{A_{\mathbf{N} / \mathbf{N}^{+}}}\right)^{1-2 S}\left\{x^{s-1}+x^{-s}\right\} d x\right) d S \\
& =c_{\mathbf{N} / \mathbf{N}^{+}} \sum_{k \geq 1} \phi_{k} \frac{1}{i \pi} \int_{\alpha-i \infty}^{\alpha+i \infty} \Gamma^{n}(S)\left(k / A_{\mathbf{N} / \mathbf{N}^{+}}\right)^{1-2 S}\left(\frac{1}{2 S-s-1}+\frac{1}{2 S+s-2}\right) d S,
\end{aligned}
$$

and the following yields (4):

$$
\begin{aligned}
h_{\mathbf{N}}^{-} & =Q_{\mathbf{N}} w_{\mathbf{N}} \Psi_{\mathbf{N} / \mathbf{N}^{+}}(1)=Q_{\mathbf{N}} w_{\mathbf{N}} \Psi_{\mathbf{N} / \mathbf{N}^{+}}(0) \\
& =Q_{\mathbf{N}} w_{\mathbf{N}} c_{\mathbf{N} / \mathbf{N}^{+}} \sum_{k \geq 1} \phi_{k} \frac{1}{i \pi} \int_{\alpha-i \infty}^{\alpha+i \infty} \Gamma^{n}(S)\left(k / A_{\mathbf{N} / \mathbf{N}^{+}}\right)^{1-2 S}\left(\frac{1}{2 S-2}+\frac{1}{2 S-1}\right) d S \\
& =Q_{\mathbf{N}} w_{\mathbf{N}} c_{\mathbf{N} / \mathbf{N}^{+}} A_{\mathbf{N} / \mathbf{N}^{+}} \sum_{k \geq 1} \frac{\phi_{k}}{k} K_{n}\left(k / A_{\mathbf{N} / \mathbf{N}^{+}}\right) \\
& =\frac{Q_{\mathbf{N}} w_{\mathbf{N}}}{(2 \pi)^{n}} \sqrt{\frac{d_{\mathbf{N}}}{d_{\mathbf{N}^{+}}}} \sum_{k \geq 1} \frac{\phi_{k}}{k} K_{n}\left(k / A_{\mathbf{N} / \mathbf{N}^{+}}\right) .
\end{aligned}
$$

Now, we prove the assertion below (5).

To start with we quote some elementary facts we will need.

1) We have

$$
P_{n}(x)=\sum_{k=0}^{n-1} \frac{1}{k !} x^{k} \leq \sum_{k=0}^{n-1} \frac{1}{k !} x^{n-1} \leq e x^{n-1} \quad(x \geq 1) .
$$

2) The derivative of

$$
g(x)=x^{\frac{2 n-2}{n}} e^{-n x^{2 / n}}
$$

is

$$
g^{\prime}(x)=\frac{1}{n}\left((2 n-2)-2 n x^{2 / n}\right) x^{\frac{n-2}{n}} e^{-n x^{2 / n}}
$$

and we have $g^{\prime}(x) \leq 0$ if $x \geq 1$ and

$$
\left|g^{\prime}(x)\right| \leq 2 x e^{-n x^{2 / n}} \quad(x \geq 1) .
$$

Moreover,

$$
g^{\prime \prime}(x)=\frac{1}{n^{2}}\left(4 n^{2} x^{4 / n}-\left(6 n^{2}-4 n\right) x^{2 / n}+\left(2 n^{2}-6 n+4\right)\right) x^{-2 / n} e^{-n x^{2 / n}},
$$

the second derivative of $g$, satisfies $g^{\prime \prime}(x) \geq 0$ if $x \geq 2^{n / 2}$. Note that (3) and (24) yield

$$
K_{n}(x) \leq 2 e n^{n-1} g(x) \quad(x \geq 1)
$$


3) If $g(x) \geq 0, g^{\prime}(x) \leq 0$ and $g^{\prime \prime}(x) \geq 0$ on $[\alpha,+\infty$, [, then $\alpha \leq a \leq b$ implies

$$
0 \leq g(a)-g(b) \leq(a-b) g^{\prime}(a) .
$$

4) If $A((n+1) / 2)^{n / 2} \geq 1$, then the derivative of

$$
h(x)=x \log ^{n}(e x) e^{-n(x / A)^{2 / n}}
$$

is

$$
h^{\prime}(x)=\left(n-\left(2(x / A)^{2 / n}-1\right) \log (e x)\right) \log ^{n-1}(e x) e^{-n(x / A)^{2 / n}}
$$

and we have $h^{\prime}(x) \leq 0$ provided that $x \geq A((n+1) / 2)^{n / 2}$ and $x \geq 1$, hence provided that $x \geq A((n+1) / 2)^{n / 2}$ if $A((n+1) / 2)^{n / 2} \geq 1$.

Now, we set $A=A_{\mathbf{N} / \mathbf{N}^{+}}, S_{n}(k)=\sum_{i=1}^{k} \frac{d_{n}(i)}{i}$ where $d_{n}(i)$ is the number of ways of writing $i$ as an ordered product of $n$ positive integers, and

$$
R_{M}=\sum_{k>M} \frac{\phi_{k}}{k} K_{n}(k / A)
$$

We want an upper bound on $R_{M}$. We note that (14) yields $\left|\phi_{k}\right| \leq d_{n}(k)$. Moreover,

$$
S_{n}(k)=\sum_{i=1}^{k} \frac{d_{n}(i)}{i} \leq\left(\sum_{i=1}^{k} \frac{1}{i}\right)^{n} \leq \log ^{n}(e k) .
$$

Thus, we have

$$
\begin{aligned}
\left|R_{M}\right| & \leq \sum_{k>M} \frac{d_{n}(k)}{k} K_{n}(k / A) \\
& \leq 2 e n^{n-1} \sum_{k>M}\left(S_{n}(k)-S_{n}(k-1)\right) g(k / A) \quad(\text { if } M \geq A)
\end{aligned}
$$

(by using (26))

$$
\begin{aligned}
& \leq 2 e n^{n-1} \sum_{k>M} S_{n}(k)(g(k / A)-g((k+1) / A)) \\
& \leq \frac{2 e n^{n-1}}{A} \sum_{k>M} S_{n}(k) g^{\prime}(k / A) \quad\left(\text { if } M \geq 2^{n / 2} A\right)
\end{aligned}
$$

(by using (27))

$$
\leq \frac{4 e n^{n-1}}{A^{2}} \sum_{k>M} k \log ^{n}(e k) e^{-n(k / A)^{2 / n}}
$$

(by using (25))

$$
=\frac{4 e n^{n-1}}{A^{2}} \sum_{k>M} h(k) \leq \frac{4 e n^{n-1}}{A^{2}} \int_{M}^{\infty} h(x) d x \quad\left(\text { if } M \geq\left(\frac{n+1}{2}\right)^{n / 2} A \geq 1\right)
$$

(by using (28)). 
Now, we set $B=(e A)^{2 / n}$ and we change the variable by setting $x=A y^{n / 2}$. We get

$$
\left|R_{M}\right| \leq 2 e\left(n^{2} / 2\right)^{n} \int_{(M / A)^{2 / n}}^{\infty} y^{n} \log ^{n}(B y) e^{-n y} \frac{d y}{y} .
$$

Since $H(y)=y^{n+1} \log ^{n}(B y) e^{-n y}$ decreases on $\left[(M / A)^{2 / n},+\infty\left[\right.\right.$ if $M \geq\left(\frac{2 n+2}{n}\right)^{n / 2} A$ $\geq e^{(n / 2)-1}$ (since its derivative

$$
H^{\prime}(y)=((n+1-n y) \log (B y)+n) y^{n} \log ^{n-1}(B y) e^{-n y}
$$

satisfies $H^{\prime}(y) \leq 0$ if $y \geq(2 n+2) / n$ and $\left.B \frac{2 n+2}{2} \geq e\right)$, we get

$$
\begin{aligned}
\left|R_{M}\right| & \leq 2 e\left(n^{2} / 2\right)^{n} \int_{(M / A)^{2 / n}}^{\infty} H(y) \frac{d y}{y^{2}} \\
& \leq 2 e\left(n^{2} / 2\right)^{n} H\left((M / A)^{2 / n}\right) \int_{(M / A)^{2 / n}}^{\infty} \frac{d y}{y^{2}} \\
& =2 e\left(n^{2} / 2\right)^{n} H\left((M / A)^{2 / n}\right) /(M / A)^{2 / n},
\end{aligned}
$$

i.e., if $M \geq\left(\frac{2 n+2}{n}\right)^{n / 2} A \geq e^{(n / 2)-1}$, then we have the following explicit upper bound :

$\left|R_{M}\right| \leq 2 e\left(\frac{n^{2}}{2} G\left((M / A)^{2 / n}\right)\right)^{n} \quad$ where $G(y)=y \log (B y) e^{-y}$ and $B=(e A)^{2 / n}$.

Now, we choose $M \approx B(\mathbf{N})=A\left(\frac{\lambda}{n} \log A\right)^{n / 2}$ and note that

$$
G\left(\frac{\lambda}{n} \log A\right)=O_{n}\left(A^{-\lambda / n} \log ^{2} A\right)
$$

yields the desired result :

$$
\left|h_{\mathbf{N}}^{-}-h_{\mathbf{N}}^{-}(M)\right|=\frac{Q_{\mathbf{N}} w_{\mathbf{N}}}{2^{n} \pi^{n / 2}} A\left|R_{M}\right|=O_{n}\left(\frac{\log ^{2 n} A}{A^{\lambda-1}}\right) .
$$

\section{Proof of Proposition 3}

Let $M \geq 0$ be a given integer. Shifting the integral (2) to the left to the line $\Re(s)=-M-\frac{1}{2}$, we pick a residue at $s=1$, a residue at $s=1 / 2$, and a residue $\operatorname{Res}_{s=-m}\left(f_{n}\right)$ at each nonpositive integer $-m \leq 0$. Hence, by using $\Gamma(1 / 2)=\sqrt{\pi}$ we get

$$
K_{n}(A)=1+\pi^{n / 2} A+2 A^{2} \sum_{m=0}^{M} \operatorname{Res}_{s=-m}\left(f_{n}\right)+\frac{A^{2}}{i \pi} \int_{-M-\frac{1}{2}-i \infty}^{-M-\frac{1}{2}+i \infty} f_{n}(s) d s .
$$

Now, it is well known that for any nonnegative integer $l \geq 0$ we have

$$
\left|\Gamma\left(\frac{2 l+1}{2}+i t\right)\right|^{2}=\frac{\pi}{\operatorname{ch}(\pi t)} \prod_{k=0}^{l-1}\left|\frac{2 k+1}{2}+i t\right|^{2},
$$

where $\operatorname{ch}(x)=\left(e^{x}+e^{-x}\right) / 2$. Hence, using the functional equation $\Gamma(s) \Gamma(1-s)=$ $\pi / \sin (\pi s)$ leads to

$$
\left|\Gamma\left(-\frac{2 l+1}{2}+i t\right)\right|^{2}=\frac{\pi}{\operatorname{ch}(\pi t)} \prod_{k=0}^{l}\left|\frac{2 k+1}{2}+i t\right|^{-2},
$$

and 


$$
\begin{aligned}
\mid f_{n}( & \left.-M-\frac{1}{2}+i t\right) \mid \\
& \leq\left(\frac{\pi}{\operatorname{ch}(\pi t)}\right)^{n / 2} \frac{A^{2 M+1}}{\left(\prod_{k=0}^{M}\left|\frac{2 k+1}{2}+i t\right|\right)^{n}}\left(\frac{1}{|2 M+2+i t|}+\frac{1}{|2 M+3+i t|}\right) \\
& \leq \frac{1}{(\operatorname{ch}(\pi t))^{n / 2}} \frac{\pi^{n / 2} A^{2 M+1}}{(M ! / 2)^{n}} \frac{2}{2 M+2} .
\end{aligned}
$$

Set

$$
c_{n}=\int_{-\infty}^{+\infty} \frac{d t}{(\operatorname{ch}(\pi t))^{n / 2}}=\frac{2}{\pi} \int_{0}^{1}\left(\frac{2}{u+u^{-1}}\right)^{n / 2} \frac{d u}{u}
$$

(note that the sequence $\left(c_{n}\right)_{n \geq 0}$ decreases, that $c_{1}=\frac{4 \sqrt{2}}{\pi} \int_{0}^{1} \frac{d v}{\sqrt{v^{4}+1}} \leq 4 \sqrt{2} / \pi$ and $\left.c_{2}=1\right)$. Then,

$$
\left|\frac{A^{2}}{i \pi} \int_{-M-\frac{1}{2}-i \infty}^{-M-\frac{1}{2}+i \infty} f_{n}(s) d s\right| \leq \frac{c_{n}}{\pi} \frac{\pi^{n / 2} A^{2 M+3}}{(M+1)(M ! / 2)^{n}} .
$$

Note that the greater the value of $n$, the faster the series (6) converges.

\section{Proof of Proposition 4}

We have

$$
\begin{aligned}
& \operatorname{Res}_{s=-m}\left(f_{n}\right) \\
& =-A^{2 m} \operatorname{Res}_{s=0}\left(s \mapsto \Gamma^{n}(-m+s) A^{-2 s}\left(\frac{1}{2 m+1-2 s}+\frac{1}{2 m+2-2 s}\right)\right) .
\end{aligned}
$$

If we set

$$
\Gamma^{n}(-m+s) A^{-2 s}=\sum_{i=-n}^{-1} a_{i}(m) s^{i}+O(1)
$$

then we get

$$
\operatorname{Res}_{s=-m}\left(f_{n}\right)=-A^{2 m} \sum_{i=-n}^{-1} a_{i}(m) 2^{-1-i}\left((2 m+1)^{i}+(2 m+2)^{i}\right) .
$$

Now, $\Gamma(s)=\frac{1}{s} \Gamma(s+1)$ yields

$$
\left.\sum_{i=-n}^{-1} a_{i}(m+1) s^{i}+O(1)=(-1)^{n}(m+1-s)^{-n}\left(\sum_{j=-n}^{-1} a_{j}(m) s^{j}+O(1)\right)\right)
$$

and

$$
(m+1-s)^{-n}=\frac{1}{(m+1)^{n}} \sum_{k=0}^{n-1} C_{k+n-1}^{n-1} \frac{s^{k}}{(m+1)^{k}}+O\left(s^{n}\right)
$$

yields

$$
a_{i}(m+1)=\frac{(-1)^{n}}{(m+1)^{n}} \sum_{j=-n}^{i} \frac{a_{j}(m)}{(m+1)^{i-j}} C_{i-j+n-1}^{n-1} .
$$

Thus, in order to simplify the recursion relation (36), we define

$$
h_{i}(m)=(-1)^{n m}(m !)^{n} a_{i}(m) .
$$


Then, using (35) yields (8), and using (34) and (36) yields (9). Note that (10) makes it easy to compute the numerical values of the $h_{i}$ 's by using Maple, for example.

\section{EXAMPLES OF RELATIVE CLASS NUMBERS COMPUTATIONS}

In order to use (4) to compute relative class numbers, it remains to explain how we compute the $\phi_{k}$ 's. Since

$$
\phi_{k}=\sum_{N_{\mathbf{N}^{+} / \mathbf{Q}}(\mathbf{I})=k} \chi_{\mathbf{N} / \mathbf{N}^{+}}(\mathbf{I})
$$

(see (14)), then $k \mapsto \phi_{k}$ is multiplicative and we only have to explain how we compute the $\phi_{p^{m}}$ where $p$ is prime and $m \geq 1$. We will only explain this when $\mathbf{N}$ is normal over $\mathbb{Q}$. In that case, let $e$ and $f$ be the inertia and residual degrees of $p$ in $\mathbf{N}_{+}$. Set $g=n /(e f)$. Then in $\mathbf{N}^{+}$we have $(p)=\left(\mathcal{P}_{1} \cdots \mathcal{P}_{g}\right)^{e}$ and

$$
\chi_{\mathbf{N} / \mathbf{N}^{+}}\left(\mathcal{P}_{1}\right)=\cdots=\chi_{\mathbf{N} / \mathbf{N}^{+}}\left(\mathcal{P}_{g}\right),
$$

and we let $\epsilon_{p}$ be the common value of these $g$ symbols. Now, $N_{\mathbf{N}^{+} / \mathbf{Q}}(\mathbf{I})=p^{m}$ if and only if $\mathbf{I}=\prod_{i=1}^{g} \mathcal{P}_{i}^{e_{i}}$ with $f \sum_{i=1}^{g} e_{i}=m$. Set

$$
C_{i}^{j}=\frac{i !}{j !(i-j) !} \text {. }
$$

Since the equation $\sum_{i=1}^{g} e_{i}=K$ has $C_{K+g-1}^{g-1}$ solutions in nonnegative integers $e_{i}$, we easily get

$$
\phi_{p^{m}}= \begin{cases}0 & \text { if } f \text { does not divide } m, \\ \epsilon_{p}^{k} C_{k+g-1}^{g-1} & \text { if } f \text { divides } m \text { and } m=k f .\end{cases}
$$

This formula (37) makes it easy to compute the $\phi_{p^{m}}$. We refer the reader to [Lou 1], [Lou 2], [Lou 3], [Lou-Oka] and [LOO] for actual computations of relative class numbers.

\section{REFERENCES}

[Lou 1] S. Louboutin, Calcul des nombres de classes relatifs: application aux corps octiques quaternioniques à multiplication complexe, C. R. Acad. Sci. Paris 317 (1993), 643-646. MR 94j: 11111

[Lou 2] S. Louboutin, Calcul des nombres de classes relatifs de certains corps de classes de Hilbert, C. R. Acad. Sci. Paris. 319 (1994), 321-325. MR 95g:11111

[Lou 3] S. Louboutin, Calcul du nombre de classes des corps de nombres, Pacific J. Math. 171 (1995), 455-467. MR 97a:11176

[Lou-Oka] S. Louboutin and R. Okazaki, The class number one problem for some non-abelian normal CM-fields of 2-power degrees, preprint Univ. Caen 1996, to be submitted.

[LOO] S. Louboutin, R. Okazaki and M. Olivier, The class number one problem for some non-abelian normal CM-fields, to appear in Trans. Amer. Math. Soc. CMP 96:12

[Oka 1] R. Okazaki, On evaluation of L-functions over real quadratic fields, J. Math. Kyoto Univ. 31 (1991), 1125-1153. MR 93b:11154

[Oka 2] R. Okazaki, An elementary proof for a theorem of Thomas and Vasquez, J. Nb. Th. 55 (1995), 197-208. MR 96m:11099

[Shin] T. Shintani, On evaluation of zeta functions of totally real algebraic number fields at non-positive integers, J. Fac. Sci. Univ. Tokyo 23 (1976), 393-417. MR 55:266

[Wa] L. C. Washington, Introduction to Cyclotomic Fields, Grad. Texts Math. 83, SpringerVerlag. MR 85g:11001

Université de Caen, U.F.R. Sciences, Département de Mathématiques, Esplanade de la Paix, 14032 Caen Cedex, France

E-mail address: loubouti@math.unicaen.fr 